\title{
Pregnancy-induced hypertension (Clinical case)
}

\author{
Hipertensão induzida pela gravidez (Caso clínico) \\ Bruno Sousa ${ }^{1,2}$, Nelson Tavares ${ }^{{ }^{*}}$
}

\begin{abstract}
${ }^{1}$ CBIOS - Research Center for Biosciences and Health Technologies, Universidade Lusófona, Av Campo Grande, 376, 1749-024, Lisboa, Portugal; ${ }^{2}$ Health Service of Autonomous Region of Madeira, Funchal, Madeira

* corresponding author: nelson.tavares@ulusofona.pt
\end{abstract}

Received / Recebido: 27/06/2020 Accepted / Aceite: 28/07/2020 Electronic Edition: www.alies.pt

A pregnant 27-year old woman, a human resources technician, with recently diagnosed high blood pressure. She was followed by the family doctor and obstetrician. She was sent to the Nutrition Consultation for overweight and pregnancy-induced hypertension. Blood pressure was monitored at home.

Keywords: Pregnancy, induced hypertension, calcium, energy

\section{Obstetric data}

Gestational age: 21 weeks and 1 day

Obstetric index: 0-0-0-0

\section{Anthropometric assessment}

Current weight: $103.3 \mathrm{~kg}$

Height: $169 \mathrm{~cm}$

Pre-conception weight: $95 \mathrm{~kg}$

BMI preconception: $33.3 \mathrm{~kg} / \mathrm{m}^{2}$

\section{Analytical Parameters (from the 1st trimester)}

\section{Hematology}

Leukocytes: $13.210^{\wedge} 3 / \mu \mathrm{L}(4.5-110)$

Erythrocytes: $5.210^{\wedge} 6 / \mu \mathrm{L}(3.80-5.80)$

Hemoglobin: $15.3 \mathrm{~g} / \mathrm{dL}(11.5$ - 16.0)

Hematocrit: $45.7 \%(37.0-47.0)$
Mulher de 27 anos, técnica de recursos humanos, grávida com hipertensão arterial diagnosti-cada recentemente na gravidez. É acompanhada pelo médico de família e pelo obstetra. Foi enviada à Consulta de Nutrição por excesso de peso e hipertensão induzida pela gravidez. Faz monitorização da pressão arterial no domicílio.

Palavras-chave: Hipertensão induzida, gravidez, cálcio, valor energético

\section{Dados obstétricos}

Idade gestacional: 21 semanas e 1 dia

Índice obstétrico: 0-0-0-0

\section{Avaliação antropométrica}

Peso atual: $103.3 \mathrm{~kg}$

Estatura: $169 \mathrm{~cm}$

Peso preconcepção: $95 \mathrm{~kg}$

IMC preconcepção: $33.3 \mathrm{~kg} / \mathrm{m}^{2}$

\section{Parâmetros analíticos (do $1^{\circ}$ trimestre)}

\section{Hematologia}

Leucócitos: $13.210^{\wedge} 3 / \mu \mathrm{L}(4.5-110)$

Eritrócitos: $5.210^{\wedge} 6 / \mu \mathrm{L}(3.80-5.80)$

Hemoglobina: $15.3 \mathrm{~g} / \mathrm{dL}(11.5-16.0)$

Hematócrito: $45.7 \%(37.0-47.0)$ 


\section{Biochemistry}

Glucose: $90.0 \mathrm{mg} / \mathrm{dL}(74.0$ - 110.0)

Urea: $23.4 \mathrm{mg} / \mathrm{dL}(8.0$ - 50.0)

Creatinine: $0.63 \mathrm{mg} / \mathrm{dL}(0.70-1.20)$

Uric acid $3.8 \mathrm{mg} / \mathrm{dL}(2.6-8.0)$

Free T4: $1.2 \mathrm{ng} / \mathrm{dL}(0.6-1.7)$

TSH: $4.86 \mu \mathrm{UI} / \mathrm{mL}(0.30-4.70)$

Beta HCG: $73821.0 \mathrm{mUI} / \mathrm{mL}$

HBsAg: negative

AcHBs: $25.33 \mathrm{mUI} / \mathrm{mL}$ (positive)

\section{Clinical evaluation}

Personal background

Obesity

\section{Family history}

Arterial hypertension: mother

Type 2 diabetes mellitus: mother and paternal grandfather

Thyroid pathology (hypothyroidism): mother

Stroke: grandfather and grandmother

Leukemia: aunt

\section{Medication}

Methyldopa $250 \mathrm{mg}$

Food Supplement (Fish oil (500 mg/g DHA Omega-3); Ascorbic acid; Ferrous fumarate; Mono and diglycerides of fatty acids; Magnesium oxide; Nicotinamide; Zinc oxide; Yellow beeswax; Lecithin; Pantothenate calcium; Pyridoxine hydrochloride; Cyanocobalamin; Thiamine nitrate; Riboflavin; Folic acid; Potassium iodide; Cholecalciferol; Biotin; Sodium selenite

Has edema of the lower and upper limbs

Denies food allergies or intolerances

Intestinal transit: regular

Has dyspepsia since early pregnancy

\section{Complementary diagnostic tests}

Obstetric ultrasound (1st trimester):

Single fetus with good vitality

Skull caudal length: $69 \mathrm{~mm}$

Biparietal diameter: $25 \mathrm{~mm}$

Nape translucency: $1.4 \mathrm{~mm}$

Nose bones present

Venous ductus: normal

Tricuspid valve: normal

Gestational age: 13 weeks and 1 day

\section{Bioquímica}

Glicose: $90.0 \mathrm{mg} / \mathrm{dL}(74.0-110.0)$

Ureia: $23.4 \mathrm{mg} / \mathrm{dL}(8.0-50.0)$

Creatinina: $0.63 \mathrm{mg} / \mathrm{dL}(0.70-1.20)$

Ácido úrico $3.8 \mathrm{mg} / \mathrm{dL}(2.6-8.0)$

T4 livre: $1.2 \mathrm{ng} / \mathrm{dL}(0.6-1.7)$

TSH: $4.86 \mu \mathrm{UI} / \mathrm{mL}(0.30-4.70)$

Beta HCG: $73821.0 \mathrm{mUI} / \mathrm{mL}$

AgHBs: negativo

AcHBs: $25.33 \mathrm{mUI} / \mathrm{mL}$ (positivo)

\section{Avaliação clínica}

Antecedentes pessoais

Obesidade

Antecedentes familiares

Hipertensão arterial: mãe

Diabetes méllitus tipo 2: mãe e avô paterno

Patologia da tiroide (hipotiroidismo): mãe

Acidente vascular cerebral: avô e avó

Leucemia: tia

Medicação

Metildopa $250 \mathrm{mg}$

Suplemento Alimentar (Óleo de peixe $(500 \mathrm{mg} / \mathrm{g}$ de DHA Omega-3); Ácido ascórbico; Fumarato ferroso; Mono e diglicéridos de ácidos gordos; Óxido de magnésio; Nicotinamida; Óxido de zinco; Cera de abelha amarela; Lecitina; Pantotenato de cálcio; Cloridrato de piridoxina; Cianocobalamina; Nitrato de tiamina; Riboflavina; Ácido fólico; Iodeto de potássio; Colecalciferol; Biotina; Selenito de sódio

Apresenta edema dos membros inferiores e superiores Nega alergias ou intolerâncias alimentares

Trânsito intestinal: regular

Apresenta dispepsia desde o início da gravidez

\section{Exames complementares de diagnóstico}

Ecografia Obstétrica ( $1^{\circ}$ Trimestre):

Feto único com boa vitalidade

Comprimento crânio caudal: $69 \mathrm{~mm}$

Diâmetro biparietal: $25 \mathrm{~mm}$

Translucência da nuca: $1.4 \mathrm{~mm}$

Ossos próprios do nariz presentes

Ductus venoso: normal

Válvula Tricúspide: normal

Idade gestacional: 13 semanas e 1 dia 


\section{Eating habits}

Wake up at 7:30 am

Breakfast: 8 am

1 slice of flat bread with 1 slice of cheese +1 sugared plain yogurt

Morning snack: 11:30 am

1 unsweetened coffee +2 Maria cookies +1 piece of fruit

Lunch: 1:30 pm (at work)

Dish: $120 \mathrm{~g}$ of grilled or cooked meat or fish $+1 / 4$ of rice / potato / pasta dish $+1 / 4$ of salad or cooked vegetables Drink water

Afternoon snack: 4 pm

1 sugared plain yogurt +1 piece of fruit or granola or muesli

Dinner: 7:30 pm

Vegetable soup (without potatoes)

Drink water

Go to bed at 0:30

Water intake: about $2 \mathrm{~L}$ per day

Coffee consumption: 1 per day

Without alcoholic habits

She mentions smoking habits (about 10 cigarettes a day), which she reduced during pregnancy.

\section{Environment, behavior and social}

She lives with her partner, and in the current pandemic situation she just keeps in touch with her family. She ate most meals at work (hotel) but has recently been on sick leave and cooks her meals at home. She says that does not add much salt in cooking. She had a sedentary activity but has already started daily walks, as indicated by the obstetrician.

\section{Questions}

1. Hypertensive disorders of pregnancy include induced hypertension, generally defined as new-onset hypertension arising after 20 weeks' gestation. Why this disorder is so important?

2. Could diet play a role in the etiology of pregnancyinduced hypertension?

\section{Hábitos alimentares}

Acorda às $7 \mathrm{~h} 30$

Pequeno-almoço: $8 \mathrm{~h}$

1 fatia de pão de forma com 1 fatia de queijo +1 iogurte natural açucarado

Lanche a manhã: 11 h30

1 café sem açúcar +2 bolachas Maria +1 peça de fruta Almoço: $13 \mathrm{~h} 30$ (no local de trabalho)

Prato: $120 \mathrm{~g}$ de carne ou peixe grelhado ou cozido $+1 / 4$ do prato de ar-roz/batata/massa $+1 / 4$ do prato de salada ou legumes cozidos

Bebe água

Lanche da tarde: $16 \mathrm{~h}$

1 iogurte natural açucarado +1 peça de fruta ou granola ou muesli

Jantar: $19 \mathrm{~h} 30$

Sopa de legumes (sem batata)

Bebe água

Deita-se às $0 \mathrm{~h} 30$

Ingestão de água: cerca de $2 \mathrm{~L}$ por dia

Consumo de café: 1 por dia

Sem hábitos etílicos

Refere hábitos tabágicos (cerca de 10 cigarros por dia), que reduziu durante a gravidez.

\section{Ambiente, comportamento e social}

Vive com o seu companheiro, e face à situação atual de pandemia apenas mantém contactos com a família. Fazia a maioria das refeições no trabalho (hotel) mas re-centemente está de baixa médica e confecciona as suas refeições em casa. Refere que não adiciona muito sal na confecção dos alimentos. Apresentava uma atividade sedentária mas já iniciou as caminhadas diárias, por indicação do obstetra.

\section{Questões}

1. Os distúrbios hipertensivos da gravidez incluem hipertensão induzida, geralmente definida como hipertensão iniciada após 20 semanas de gestação. Porque esse distúrbio é tão importan-te?

2. A dieta poderá desempenhar um papel na etiologia da hipertensão induzida pela gravidez? 
3. Could an association be found between dietary factors such as energy intake and pregnancy-induced hypertension?

4. Is calcium an important nutrient in this disorder?

\section{Authors Contributions Statement}

Both authors contributed to conceptualization and study design, data analysis, drafting, editing and reviewing, and final writing.

\section{Funding}

Not applicable

\section{Acknowledgements}

The authors would like to express their thanks to the participant in the case study.

\section{Conflict of Interests}

The authors declare there are no financial and/or personal relationships that could present a potential conflict of interests.
3. Foi encontrada associação entre factores alimentares, como ingestão de energia e hiperten-são induzida pela gravidez?

4. O cálcio é um nutriente importante nesse distúrbio?

\section{Declaração sobre as contribuições do autor}

Ambos os autores contribuíram para a conceção e desenho do estudo, análise de dadosm redação, edição e revisão, supervisão e redação final.

\section{Financiamento}

Não aplicável

\section{Agradecimentos}

Os autores desejam expressar os seus agradecimentos a participante no caso de estudo.

\section{Conflito de Interesses}

Os autores declaram que não há relações financeiras e/ou pessoais que possam repre-sentar um potencial conflito de interesses. 
$\cdot\left(\varepsilon^{6} \mathrm{I}\right)$

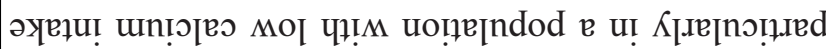

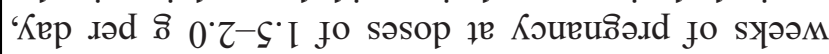

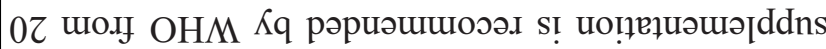

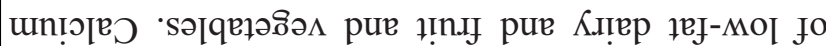

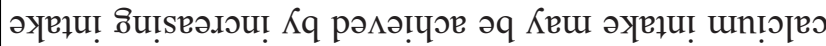

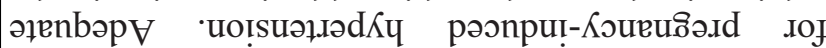

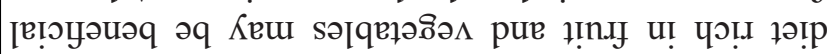

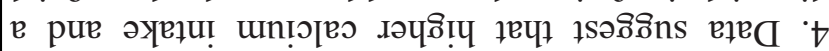

$\cdot(\varepsilon)$ uo!̣suəł.

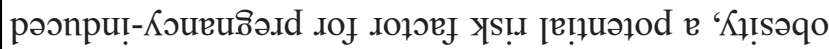

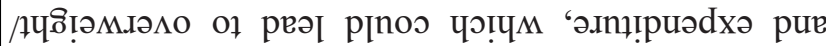

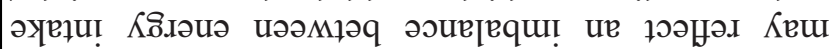

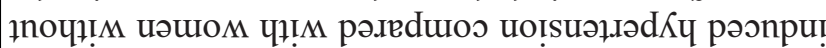

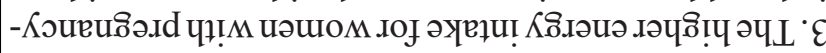

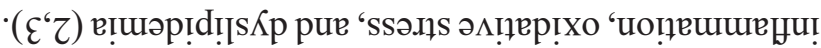

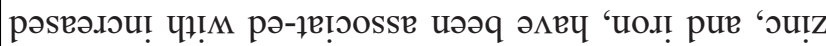

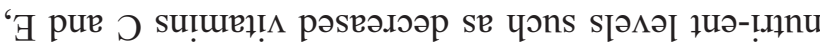

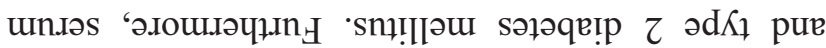

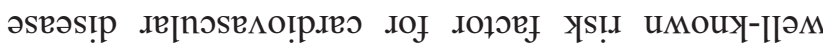

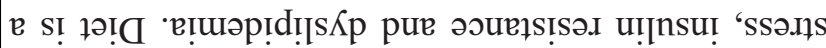

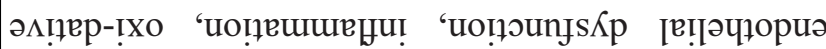

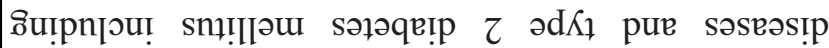
IR[nоse

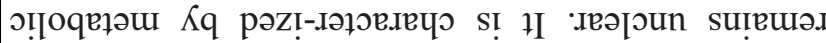

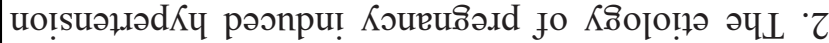

$\cdot\left(z^{6} \mathrm{I}\right)$

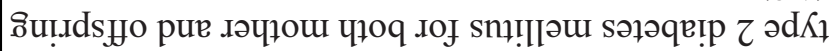

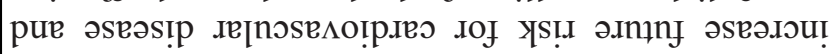

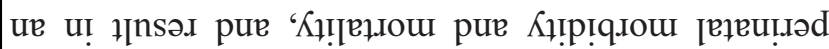

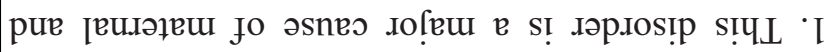

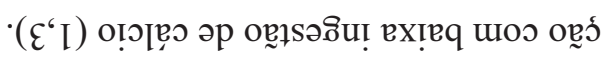

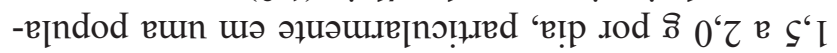
әр sәsop шә zәр!

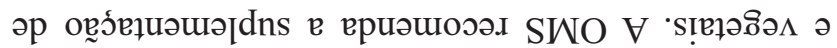

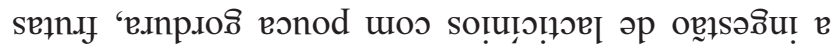

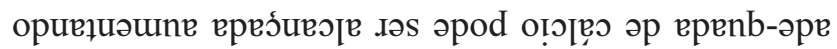

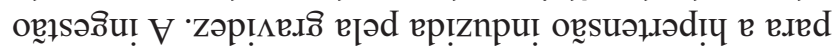

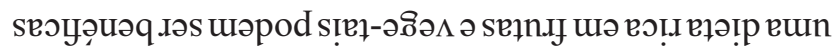

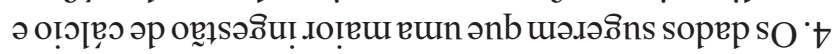

$\cdot(\varepsilon)$

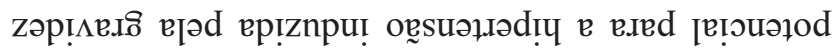

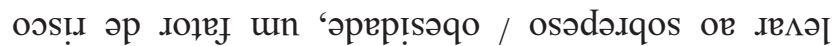

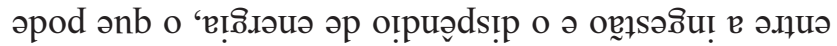

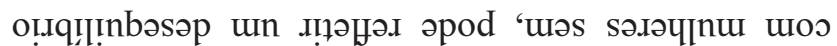

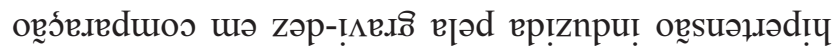

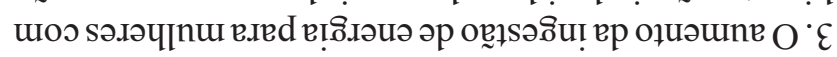

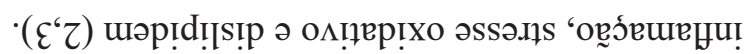

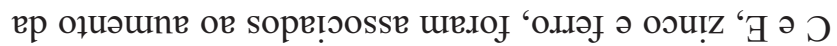

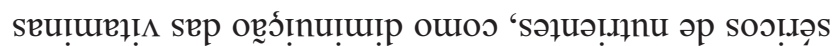

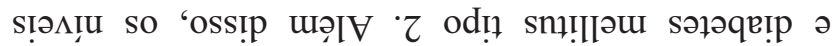

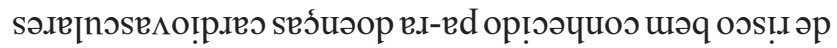

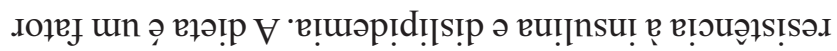

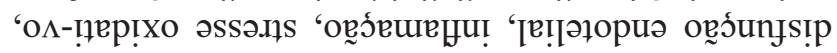

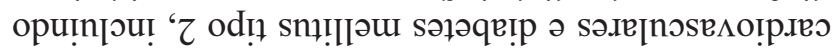

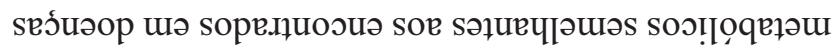

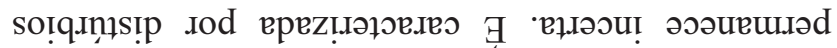

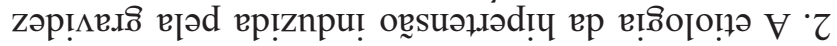

$\cdot\left(Z^{6} \mathrm{I}\right)$ оपाए ә

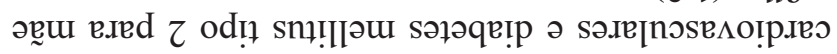

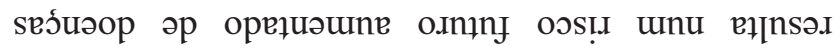

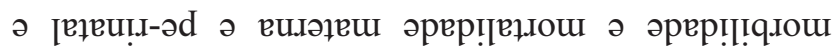

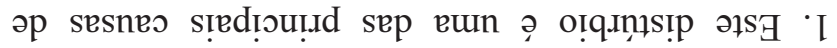




\section{References / Referências}

1. Hofmeyr GJ, Betrán AP, Singata-Madliki M, et al. Prepregnancy and early pregnancy calcium supplementation among women at high risk of preeclampsia: a multicentre, double-blind, randomised, placebo-controlled trial. Lancet. 2019; 393(10169):330-339. doi:10.1016/S0140-6736(18)31818-X

2. Kintiraki E, Papakatsika S, Kotronis G, Goulis DG, Kotsis V. Pregnancy-Induced hypertension. Hormones (Athens). 2015;14(2):211-223. doi:10.14310/ horm.2002.1582

3. Schoenaker DA, Soedamah-Muthu SS, and Mishra, GD. The association between dietary factors and gestational hypertension and pre-eclampsia: a systematic review and meta-analysis of observational studies. BMC Med. 2014; 12:157. https://doi.org/10.1186/s12916-014-0157-7 